Wren Strabucchi, Sandra Iturriaga

Edición de levantamientos: Arturo Lyon

\title{
Jardín a dos tiempos
}

Con la ciudad jardín como modelo, ciertos barrios de Santiago tienen en el jardín una pieza clave del puzzle urbano: mediador con la calle, expansión privada al interior de la manzana, articulador con el paisaje del valle y regulador de relaciones entre vecinos. Nuevas vocaciones se inauguran con la incorporación de estos espacios domésticos -abiertos y sin programa- al ámbito de lo colectivo, a través de operaciones integradoras que construyen un total atendiendo cada parte.

Palabras clave: Campus Lo Contador, levantamiento, jardines, ciudad - jardín, urbanismo.

With the garden city as their model, some Santiago neighborhoods have taken the garden as a key to the urban puzzle, as mediator with the street, private expansion within the block, link with the landscape of the valley, and regulator for neighbors' relationships. New vocations arise as these open un-programmed domestic spaces are incorporated into the collective sphere, integrated through actions that create a whole, yet take account of each part.

ardens, garden city, town planning

I. Tiempos del lugar

De los jardines de 6 casas a jardín universitario

Lo que a continuación se presenta es el registro y descripción del estado de las cosas sobre un territorio aparentemente pequeño, un jardín ${ }^{1}$, que en su proceso de cambio y transgresión en el tiempo a un orden original, posibilita múltiples lecturas de una realidad rica e intensificada. No es un proyecto, y constituye sin embargo una lección de arquitectura.

Es un hecho que desde hace una década la Facultad de Arquitectura, Diseño y Estudios Urbanos de la Universidad Católica ha venido anexando paulatinamente a su territorio original - la casa de Lo Contador- un total de 6 casas emplazadas hacia su costado oriente, y cuya extensión más importante la constituye la ex casa del arquitecto Sergio Larraín García Moreno², construida al interior de la manzana y alrededor de la cual se ubican las demás.

$\mathrm{Si}$ bien son casas que al pasar a formar parte de una comunidad universitaria son objeto de transformaciones de su estructura construida, el cambio menos evidente (y más radical) se origina en el territorio entre casas, apareciendo una condición inédita al interior de sus jardines que transgrede su orden original:

Si originalmente existía para cada casa una secuencia calle - vereda - antejardín - casa - jardín, al anexarse a un orden mayor del campus, esta secuencia se transforma en un paisaje de jardines interiores.

La condición de este paisaje-al no desaparecer del

1 Jardín, del lat. vulgar gardinus,

2 Antigua casa de inquilinos del fundo 10 Contador, que fue comprada y remodelada por Sergio Larraín a finales de la década del cincuenta. Ver p.54, ARQ 59. 3 "Perderse significa que entre nosotros y el espacio no existe solamente
una relación de dominio, de control una relación de dominio, de control
por parte del sujeto, sino también la posibilidad de que el espacio nos domine a nosotros. Son momentos de la vida en los cuales empezamos a aprender del espacio que nos rodea.

Tal como el cuento de Borges $E I$ Jrdin de los senderos que se bifurcan una trama creciente $y$ vertiginosa de tiempos, que se aproximan, se bifurcan, y se cortan abarcando todas las posibilidades, los jardines de Lo Contador constituyen en su posibilidad vida universitaria. ${ }^{5}$ Cuanto más se campus de la U. Católica, más se distingue su condición paradigmática de jardín en el que transcurre la vida del estudio. Similar si se quiere a los
jardines de los fellows en los colleges de jardines de los fellows en los colleges de su cualidad y no en su exclusividad.
Situación: en tanto sitio, lugar circunstancia, ocasión 'Levantamiento: Aiuste, conclusión y finiquito de cuentas. Digno de memoria. Abertura: grieta, hendidura. Cualidad que el sonido recibe según sea la

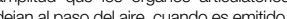
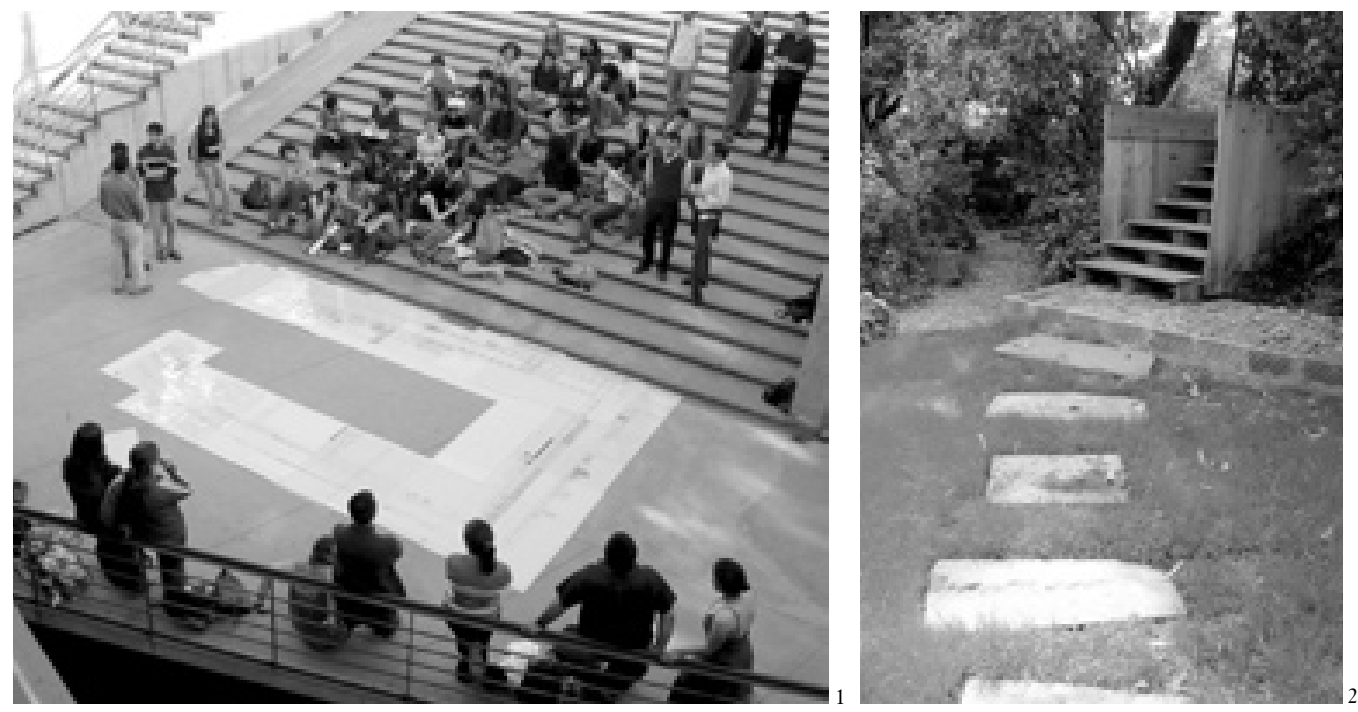

todo la estructura de los medianeros existentesacentúa una secuencia de jardines como un laberinto en el que aún es posible perderse ${ }^{3}$, un itinerario de senderos que se bifurcan entre fragmentos de piezas memorables, secuencias de umbrales y claros, y pasos discontinuos entre casas que se vuelven pabellones ${ }^{4}$.

Si bien al integrarse a la vida universitaria estos jardines asumen una condición más pública, aún mantienen ese carácter de intimidad y domesticidad de jardines de casas, al punto que al recorrerlos surge la duda sobre el traspaso de propiedades privadas, al modo como se cruza un vallado en el campo como un acontecimiento inesperado ${ }^{5}$.

No es un área verde, es un jardín. Un recinto amurallado que se presenta en una permanente tensión entre un orden determinado y un orden azaroso, un territorio domesticado y un campo de juego en continua transformación, una realidad que las más de las veces sólo el tiempo es capaz de imprimir como cualidad a las obras proyectadas.

\section{Tiempos del registro.}

Levantamiento de una situación

Durante cuatro semestres consecutivos el Taller de Formación de II semestre de la EAUC se ha dedicado a registrar la situación ${ }^{6}$ de estos jardines anexados, haciendo del levantamiento ${ }^{7}$ exhaustivo de ellos una materia relevante de estudio. Dos puntos han orientado este quehacer:

La situación cotidiana como un hecho memorable ${ }^{8}$ : El reconocer aquellos hechos de nuestro entorno cotidiano con los cuales uno convive como un territorio rico y complejo, y que paradójicamente al estar cercano, pareciera ocultar su interés a los arquitectos como materia de reflexión para la disciplina.

El registro de una situación cotidiana: La tarea de levantamiento y registro exhaustivo de una realidad concreta representa un modo de desocultar un orden, que la situación en su cotidianeidad oculta con mayor insistencia. El registro y sus posibles lecturas permiten verificar cómo este entorno queda permeado por una permanente relación entre forma de vida y forma construida, presentando adecuaciones y transgresiones a un orden original a partir de determinadas situaciones y formas de ocupación en el tiempo.

Estos levantamientos posibilitan por tanto una base para trabajar sobre la tensión de una descripción de las cosas como son y la descripción de las cosas como pueden ser, esto es, un acercamiento a la riqueza de la realidad como un fenómeno susceptible de ser pensado, representado, pero no controlado en su totalidad.

Pasos entre piezas memorables

La abertura de los jardines como transgresión de los medianeros 9

Si bien las seis casas adquiridas por la Universidad se han ido anexando paulatinamente en el tiempo, los jardines irrumpen dentro del ritmo de la vida universitaria desde el momento en que se producen aperturas puntuales sobre sus medianeros, abriendo 
${ }^{10}$ Apertura: inauguración, inicio, comienzo. 11. "Un día los hombres des alfabeto en los ojos de las calcedon en los pardos terciopelos de la falena y entonces se sabrá con asombro qu cada caracol manchado era, deste en Los pasos perdidos.
12 "...) muy a menudo el errar podría ser considerado como un valor más que el andar es un instrumento capaz de describir y de modificar aquellos espacios metropolitanos que a menudo presentan una naturaleza que deberi comprenderse y llenarse de significados, mas que proyectarse
13 "Sincopado: ritmo -o secuenciapres "Shcop midos En: AA.W.: Diccionario Metápolis. Arquitectura avanzada. Ed. Actar, Barcelona, 2001. "Los bolies o "capillas" en los juegos
1 Montaje de los levantanientos, taller de II semestre, EAUC

2 Puente escalera entre la cas SLGM y SEREX. Curso Introducción a la Técnica, EAUC

3 Patio casa SLGM

4 Senderos interiores en ardines, campus Lo Contador

5 Huellas del medianero entre el patio de acceso a la casa SLGM el patio de esculturas de
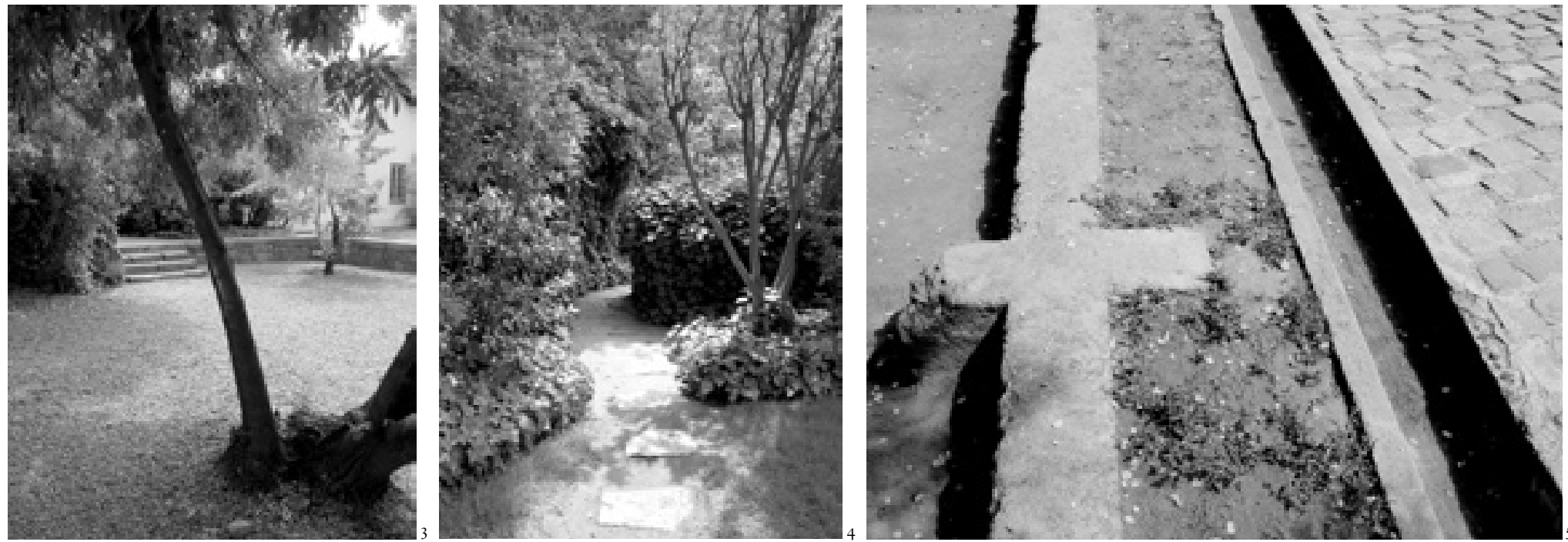

paso a una secuencia de piezas memorables desde el interior del Campus y con ello a un tiempo no imaginado ni aún planificado.

Esta transgresión al orden original de los medianeros como separación entre solares, se ha dado desde tres dimensiones de apertura:

a. Lo abierto entendido desde una condición de puerta que media entre dos realidades distintas, manteniendo temporal o permanentemente relaciones de intercambio (puerta entre el jardín principal de la ex casa de Sergio Larraín y Ediciones ARQ, y entre el jardín del Doctorado y el del Magister). b. Lo abierto como posibilidad de un acontecimiento inesperado que irrumpe al caer un muro parcial o totalmente (patio posterior de esculturas de la ex Escuela de Arte, que se vuelve una antesala hacia el acceso de la ex casa de Sergio Larraín al caer el muro de adobe que los separaba).

c. Lo abierto como inauguración de una medida a escala de Campus, al multiplicar los itinerarios que posibilitan un reconocimiento de sus territorios (ejemplo de esto es la escalera propuesta por los alumnos del curso Introducción a la Técnica para salvar la diferencia de nivel y accesibilidad entre la ex casa de Sergio Larraín y la casa ocupada por SEREX).

La apertura de los jardines como secuencia de piezas y umbrales ${ }^{10}$

La representación convencional en planta oculta la relación más cierta entre un jardín y otro, por cuanto lejos de representar un conjunto unitario, los jardines se ensamblan en torno a umbrales y secuencias de traspaso que acentúan su condición de piezas autónomas y ejemplares.

Esta secuencia de umbrales -entre aberturas de medianeros y cambios en el relieve del suelopivotea principalmente en torno a la ex casa de Sergio Larraín y sus jardines, que al anexarse al orden más extenso del campus intensifica su condición existente de pieza ejemplar: pocas veces somos más conscientes de la cualidad de subir a un jardín como piso noble que en las simples gradas que separan la actual cancha de fútbol con el jardín principal de la casa, y pocas veces percibimos la cualidad estructurante de un medianero como pieza ejemplar como en la pirca que separa la casa hacia el sur del resto de las casas de la manzana.

Piezas de un itinerario memorable

El jardín de los pasos perdidos ${ }^{11}$

A partir de la conexión -existente o posible- de los jardines, ya no sólo se está en ellos, sino que además se los atraviesa.

La condición particular de este atravieso queda dada por el cruce a través de piezas y huellas de pavimento que aquilatan la memoria de un tiempo doméstico, pero que al recorrerse como parte del presente de la vida universitaria, irrumpen como una nueva narración de significación colectiva: las terrazas, galerías y piscinas, pasan así de ser piezas de estancia privada a piezas de un itinerario atravesable.
Su alternancia casual y espontánea entre jardines, secuencian un andar errático ${ }^{12}$ y asincopado ${ }^{13}$, que está cerca de permitirnos descubrir nuestro propio bolie en este lugar ${ }^{14}$.

\section{El antejardín de los pasos encontrados}

Por otro lado, a partir de la conexión -existente o posible- de los antejardines de las casas, se posibilita que ya no sólo se transite en un recorrido continuo a través de la vereda, sino que se esté en ella.

$\mathrm{Al}$ no existir ya un ritual doméstico que requiera de la autonomía de cada antejardín, el recorrido lineal y regular de la vereda, en su vecindad y conexión a éstos, posibilita la cualidad de pieza urbana a modo de una ribera de río con múltiples meandros, un recorrido tramado entre los tiempos del cauce y detención de la vida urbana en su cruce con la vida universitaria.

La vereda es a los antejardines, lo que el recorrido interior de los jardines es a las piezas de pavimento: continuidad que posibilita el ensamblaje entre piezas descalzadas. Un descalce en el tiempo que enriquece la trama de acontecimientos de nuestra vida cotidiana. ARQ

Bibliografía: La Cecla, Franco; Perdersi, l'uomo senza ambiente. Ed. Laterza, Bari, 2000. / Careri, Francesco; Walkscapes. El andar como práctica estética, Ed Gustavo Gili, Barcelona, 2002. 


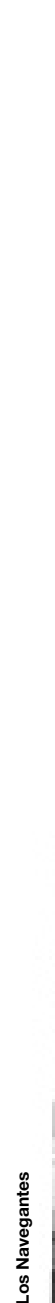



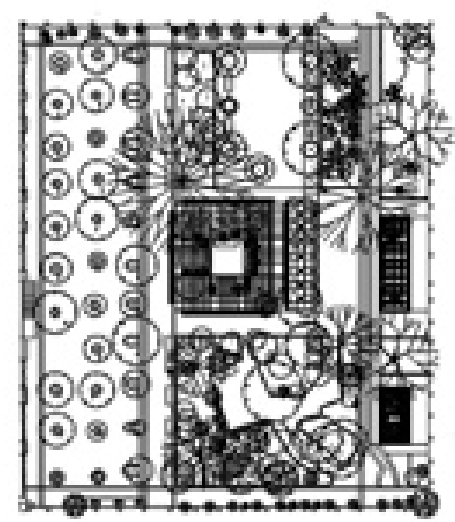

A Jardín de 6 casas y su anexión en el tiempo.

En base a levantamiento realizado por los alumnos del

taller de arquitectura de II

semestre, primavera de 2003, a

cargo de los Prof. Pedro Alonso,

Sandra Iturriaga, Marcelo Sarovic

y Wren Strabucchi.

Data de las escrituras de compra

de las casas proporcionada por

Marcos Bravo

1 El Comendador 1946 - ex Casa Sergio Larraín García Moren

(25.01.01)

2 El Comendador 1954 - sede

3 EI Comendador 1966 - sede

Publicidad Universitaria

(21.01.99)

4 El Comendador 1968 - sede

Magister Arq. + I.E.U. (22.12.99)

5 El Comendador 1970 - sed

6 Los Navegantes 1963 - sede

B Patio y jardín Casa Lo Contador En base a levantamiento realizado por los a lumnos del taller de I año pel postumnos del taller de del paisaje, primavera de 2000

cargo de los profesores

Mario Pérez de Arce y

Juan Cristóbal Edwards 


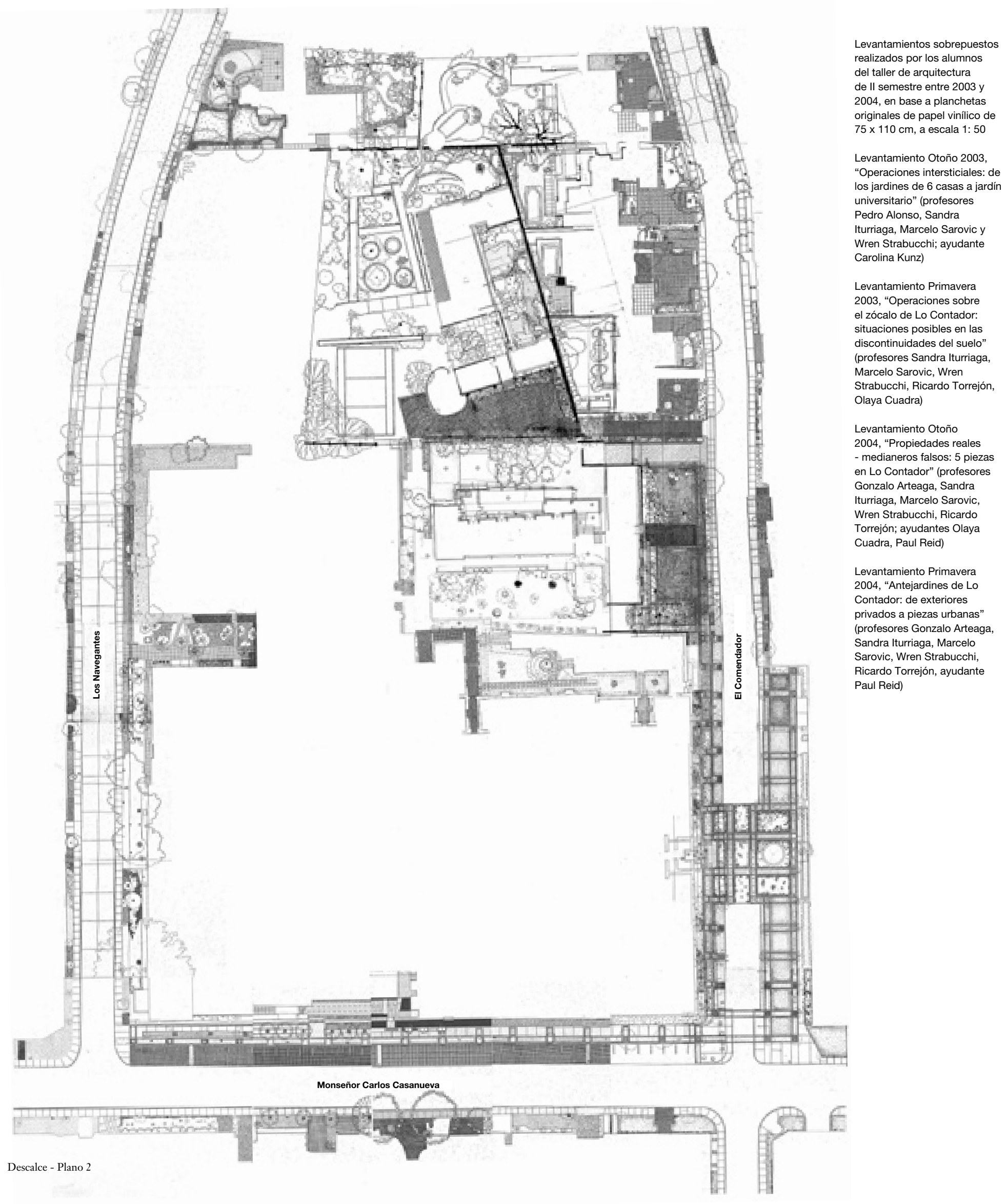




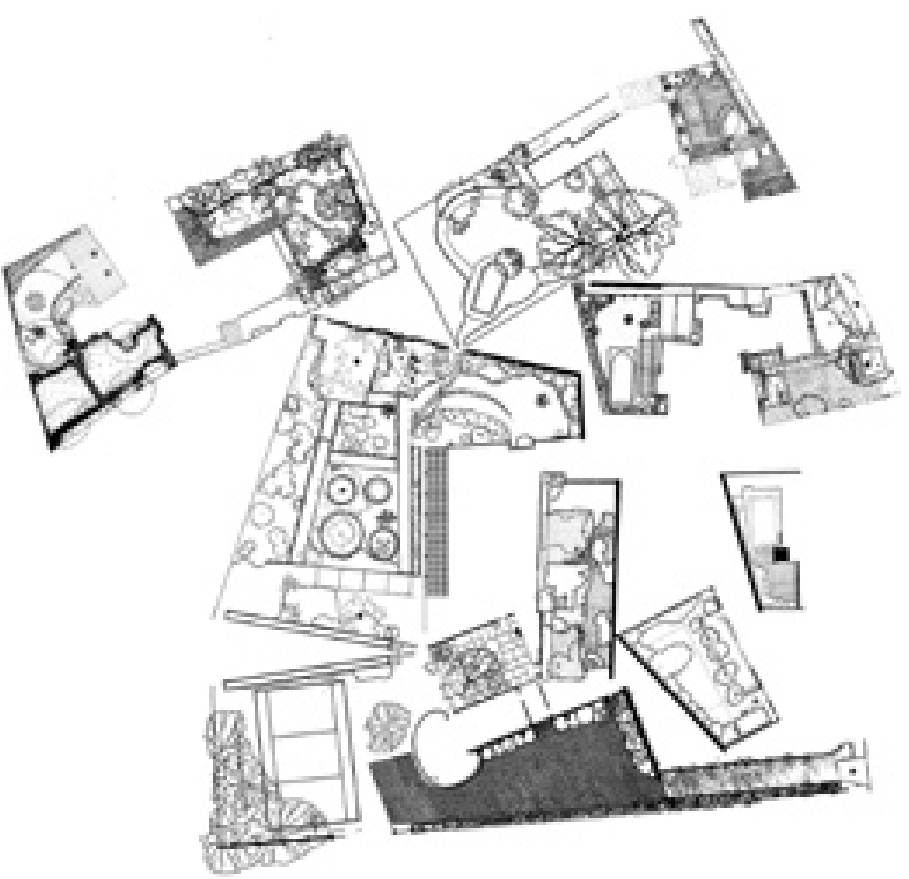

Piezas y umbrales - Plano 3

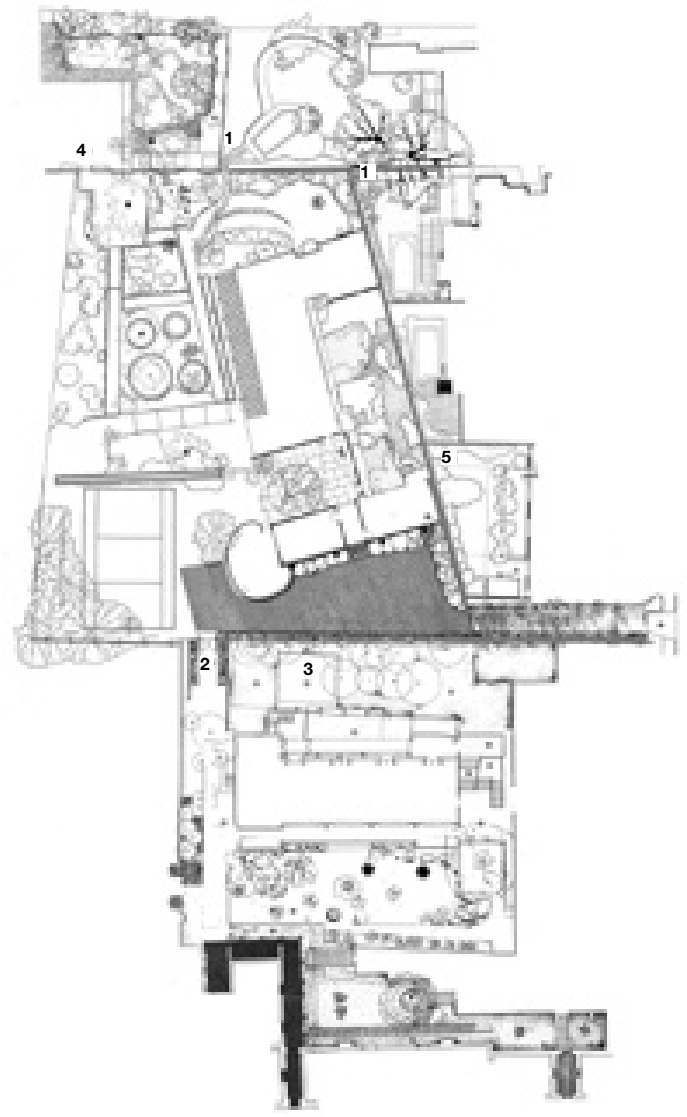
Aberturas existentes entre
medianeros a la compra de las casas

Se abre reja en febrero de Arquitectura y los jardines de la casa de Sergio Larraín

3 Se cae el muro de adobe en invierno de 2002, conectando patio de la ex Escuela de Arte Tergio Larrí

Se abre reja en febrero de 2003 desde los jardines Sergio Larrain a Ediciones ARQ

5 Se construye puente-escalera

Jilo de 2004 , ejercicio realizado

Introducción a la Técnica a

de los profesores Claudio Vásquez

con el taller de II semestre, ri:
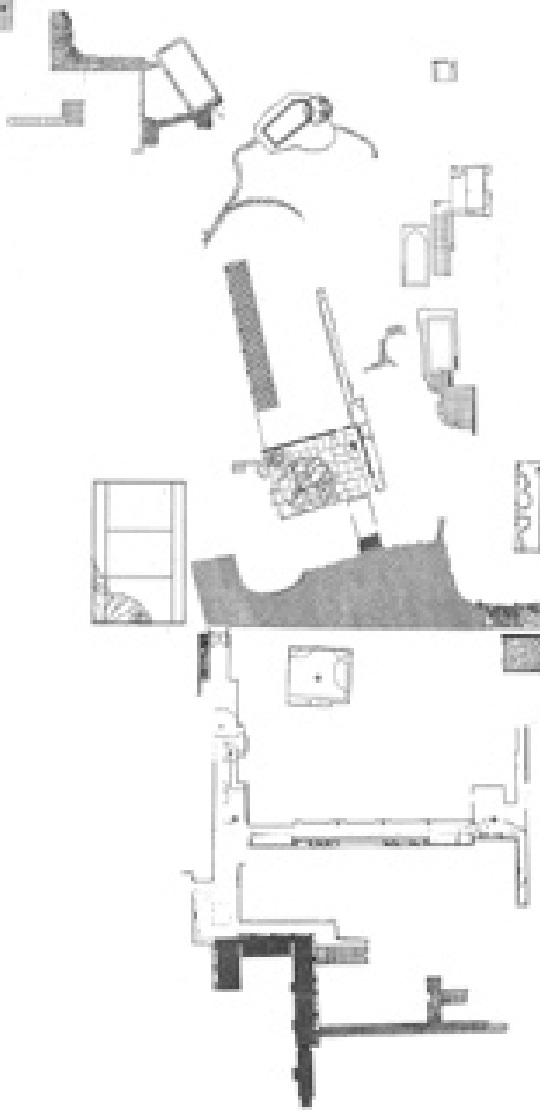

3)

isting

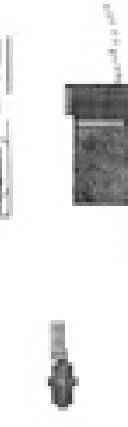

Medianeros y aperturas - Plano 4 

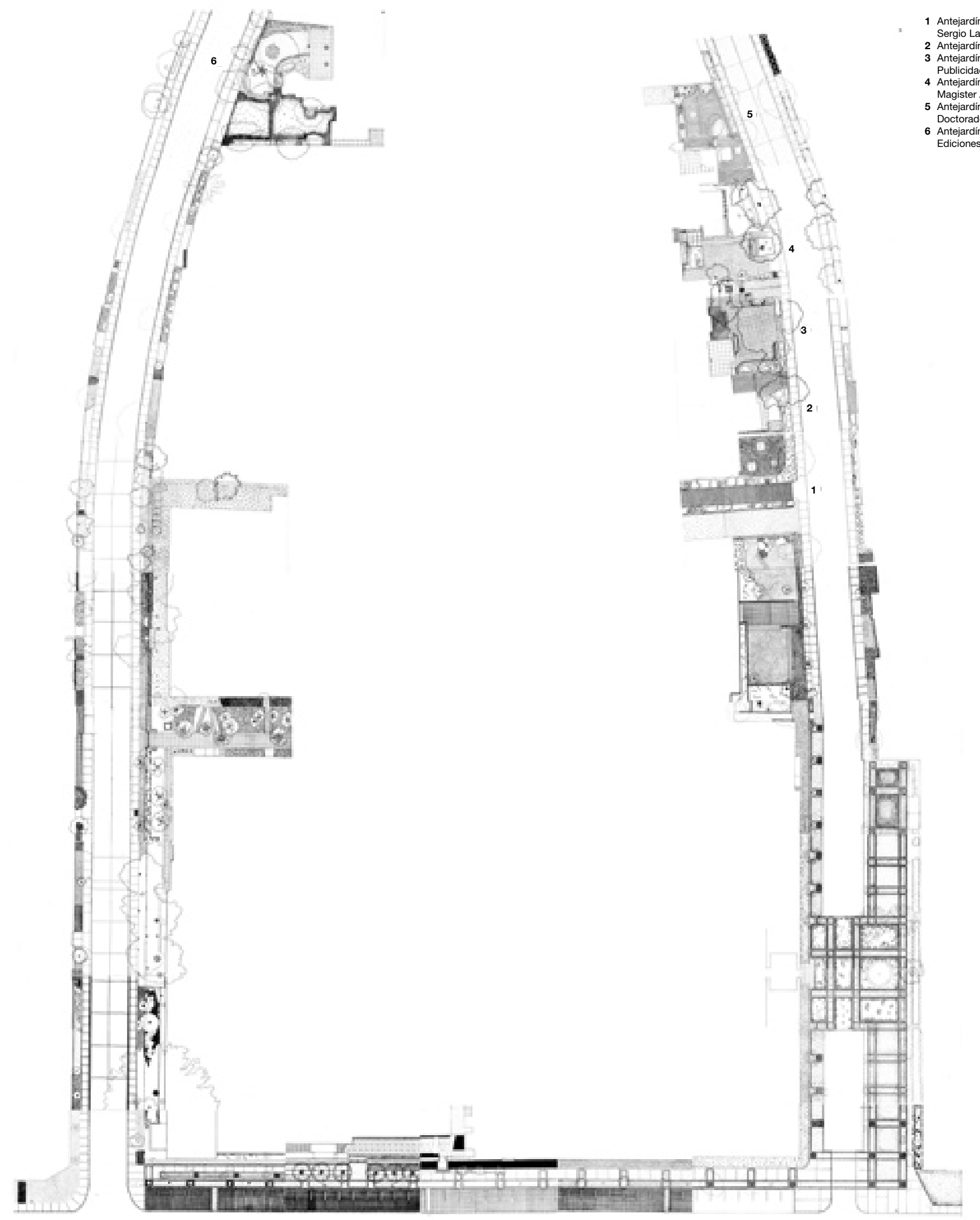

Sergio Larraín García Moreno

Antejardín casa sede 